\title{
Modeling Portable Manager Aiding In the MANET Communication
}

\author{
Ganesh Kumar, A. Arivazhagan \\ AMET University, Chennai, India
}

\section{Article Info}

Article history:

Received Nov 21, 2017

Revised Jan 29, 2018

Accepted Feb 17, 2018

\section{Keywords:}

Delay

Loss

Mobile ad hoc network

Portable Manager

Packet received

\begin{abstract}
The aim of this paper is to model the Portable Manager and allow it to aid in communication among Mobile Ad-hoc Networks (MANETs) carrying information across the mobile nodes in the network. In this article, Modeling Portable Manager aiding in the MANET Communication (MMAC) is proposed. The novelty is present in the use of node performance parameter called as $\alpha$ defined as the ratio of the packet received, packet loss and delay of the node which could be used for the next hop selection in the mobile ad hoc network. The Portable Manager (PM) is used to find out the relay node in the network. The working of the PM with a minimal number of nodes is analyzed and presented through the simulations in the network simulator.
\end{abstract}

Copyright $\odot 2018$ Institute of Advanced Engineering and Science. All rights reserved.

\section{Corresponding Author:}

Ganesh Kumar,

AMET University, Chennai,

India.

\section{INTRODUCTION}

A MANET contains number of movable nodes. These movable nodes are able to transmit the data to every node without the assistance of base stations. Quality of Service (QoS) prerequisite, for example, those for multimedia applications with transfer speed and vitality imperative has been seriously considered as of late as an issue for MANETs. Node Movement and wireless radio properties make it more difficult to provide quality of service in MANETs. Figure.1 shows the example scenario of MANET.

Therefore, in this article, Modeling PM aiding in the MANET Communication is proposed. MA is a programming entity that follows up for the benefit of their creators and move independently between nodes. In general, a PM executes on a machine that ideally provides the resources or services that it needs to do its works.

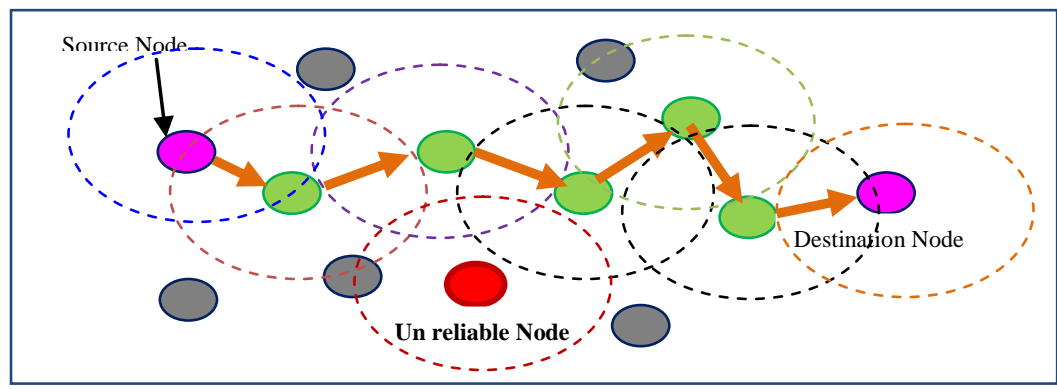

Figure 1. Example MANET Scenario 


\section{RELATED WORKS}

Productive asset disclosure and ordering is the guaranteed approach to give minimal effort altered asset recovery benefit in a flexible specially appointed condition. Routing Intelligent Mobile Agents [1] frequently gather availability of resources, routing and index. In [2] analyzed the node capacity and delay in MANETs. It received optimal scheduling parameters. A cross layer approach had proposed to utilize the measured SNR value on the physical layer as a routing metric. The proposed technique has the advantage of providing a good quality measurement to the link without any additional traffic required. The proposed technique is used in the Dynamic Sequence Routing Protocol (DSDV) module [3].

A dynamic computation using distributed algorithm for minimum hop paths is proposed; here Bellman Ford algorithm is added with distributed algorithm for the determination of shortest path computation. In this algorithm, each node maintains its next hop i.e. successor node and for each destination in the network the shortest distance in number of hops are determined. The modified algorithm would have to maintain the length and hop count of the shortest path to each network destination [4]. Distributed core selection and migration protocols for MANET's with dynamically changing network topology was presented. In static networks core selection protocols are not suitable ad hoc networks, since these algorithms depend on knowledge of whole network topology and not suitable for dynamic topology. A neighbour coverage-based probabilistic rebroadcast protocol for reducing routing overhead in MANETs is proposed. To effectively exploit the neighbour coverage knowledge a novel rebroadcast delay is proposed along with the probabilistic rebroadcast protocol which is used to determine the rebroadcast order and accurate additional coverage ratio by sensing the neighbour nodes. Connectivity factor is defined to provide the node density adaptation and the additional coverage ratio is combined in order to set reasonable rebroadcast probability [5]. The core location method implemented here is based on the notion of median node of the current multicast tree instead of the median node of the whole network and hence the multicast tree is declared as good approximation for entire network. Core-based multicast routing algorithms root the multicast tree at a specific node called the core node. The core node is also called a centre node or a rendezvous point. Distributed core selection does not require any distance information but uses the sum of weights of all the links in the tree which signifies the total bandwidth consumed for multicasting a packet [6].

The ad hoc network is self-organized by ad hoc network routing protocols. Due to the mobility of nodes, routing protocols is comprised of several direct node-to-node links exists only for a certain period. Therefore, the estimated probability density function had proposed to improve the routing performance. The exponential distribution was used to predict route's behavior. And discrete Gamma distribution is proposed to estimate time distribution [8]. Neighbour Coverage Based Probabilistic Rebroadcast (NCPR) [7] was proposed. This protocol completely relies on preset variables, which are required to be set by the system administrator based on the ad-hoc scenario. The routing overhead crisis caused by RREQ redundant packets could be overcome by applying the NCPR protocol. Based on the self-punning scheme, the number of redundant RREQ messages is reduced relying on all the nodes in the network. Basically, due to multiple varieties in node deployment, this number is insufficient when the network is congested. In [9], the quantity of clients and radio wires with the same transmitted power, the cluster pick up increments directly. The enhanced cluster pick up can be expanded under enormous MIMO framework, while the viewable pathway subsequent to expanding the quantity of reception apparatuses to $100 \mathrm{M}$ is as yet soaked. The addition of cluster pick up will enhance the QoS and scope range. Thus, the quantity of reception apparatus components on account of the viewable pathway increments, and the transmitted flag additionally increments in light of the condition of the channel demonstrate and accessible ideal transmitted power.

\section{PROPOSED WORK}

The proposed work is a simple analysis of the data that has been used by the mobile nodes to enhance communication. A performance parameter called $\alpha$ has been proposed to assess each node so that its possibility of being selected as the next node is increased.

In order to reach the source data to destination in the most Energy efficient route selection is an important process. The PM monitors the all node reliability of data communication operations and it sends the information to every node. The nodes reliability calculation depends on Packet Delivery Rate and Delay Rate and Loss rate of data transmission. The Node Reliability $(\alpha)$ computation is given.

$$
\alpha=P R R+\frac{1}{P L R}+\frac{1}{D R}
$$

Where, 
$P R R \rightarrow$ Packet Received Rate

\section{$P L R \rightarrow$ Packet Loss Rate}

$D R \rightarrow$ Delay Rate

The PM gathering all nodes $\alpha$ status and it decides which node is reliable and unreliable in MANET. Then it send notification message to all nodes. If a source desires the data to destination, it verifies the node is reliable or not then source select that node is next hop. This process is continued until the source reaches the destination.

\section{PERFORMANCE EVALUATION}

In our simulation analysis, the following metrics are used to analyze the results in MANETs.

Packet Delivery Rate (PDR): is defined as the ratio of total data packets received by the destination to total send packets by source multiplied with number of receivers. The PDR is calculated by the Equation (2).

$$
P D R=\frac{\text { Total Pack Received }}{\text { Total Pack Send }}
$$

Packet Loss Ratio (PLR): The Packet Loss Rate (PLR) is the ratio of the number of packets dropped to the number of data packets sent. The PLR is calculated by Equation (3).

$$
P L R=\frac{\text { Total Pack Dropped }}{\text { Total Pack Send }}
$$

Delay: It is defined as the average time that a packet takes to transmit the network from source to destination. It is measured by Equation (4).

$$
\text { Delay }=\frac{\text { Pack RecvdTime }- \text { Pack Sent Time }}{\text { time }}
$$

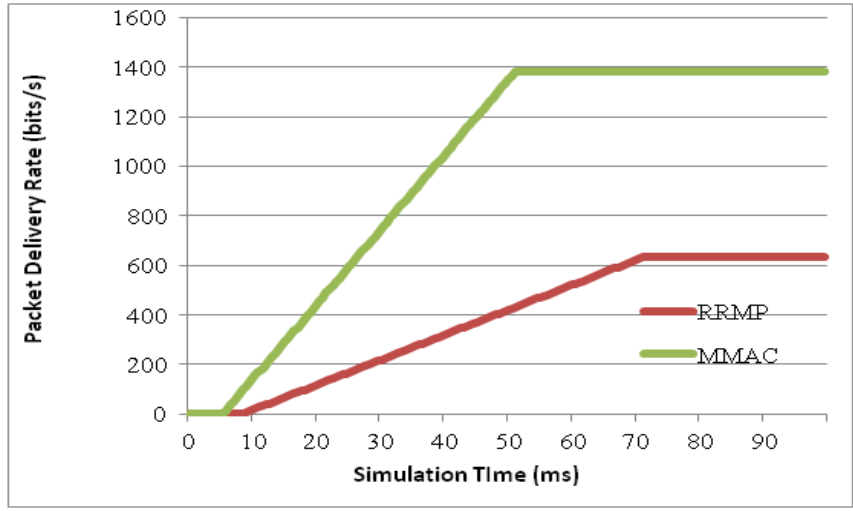

Figure 2. Packet Delivery Rate

From Figure 2, the proposed protocol MMAC that increases the packet received rate compared to the existing protocol RRMP. The Figure 3 indicates the packet loss rate of the proposed protocol MMAC is lesser than the RRMP protocol showing the efficiency of the MMAC. 


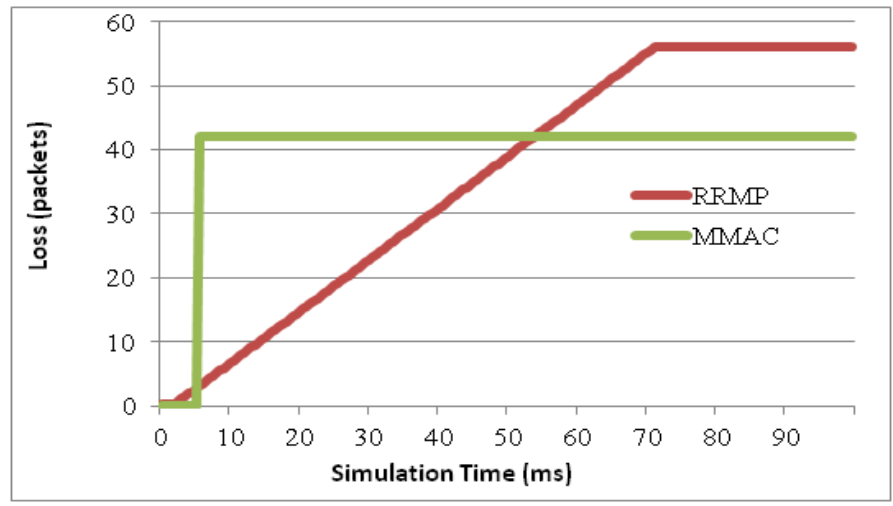

Figure 3. Packet Loss Rate

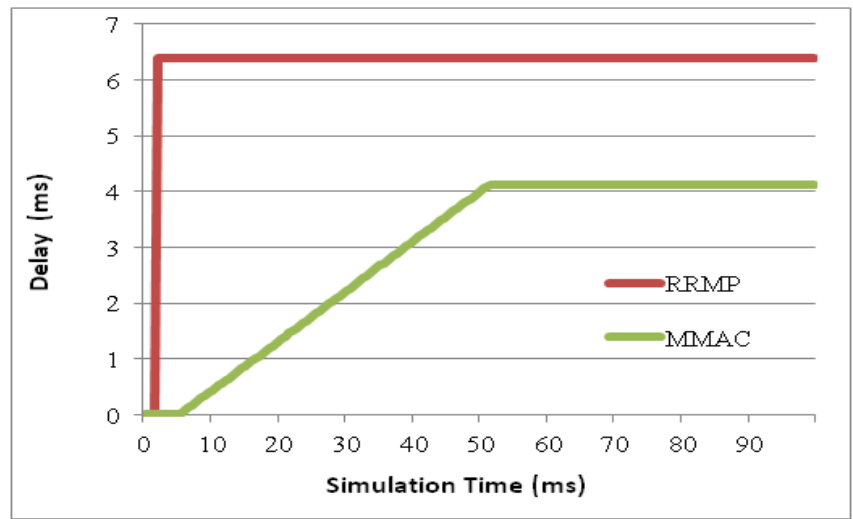

Figure 4. Average Delay

Figure 4 demonstrate that the delay of RRMP and MMAC. The average delay of the RRMP is larger than the MMAC indicating the improved performance of the MMAC protocol.

\section{CONCLUSION}

In this scheme, the Portable Agent checks the mobile node reliability. Thus the source transmits the data to reliable node. This scheme reduces both the delay of network and control message overhead in the network. This scheme provides better routing stability and it reduces the link breakage. In addition this scheme meets the QoS requirement in MANET. The simulation result demonstrate that the improve packet delivery rate and reduce the latency in the network.

\section{REFERENCES}

[1] Jia R, Yang F, Yao S, Tian X, Wang X, Zhang W, Xu J. Optimal Capacity-Delay Tradeoff in MANETs With Correlation of Node Mobility. IEEE Transactions on Vehicular Technology. 2017; 66(2); 1772-1785.

[2] Valera, Alvin C, Winston Khoon Guan Seah, S V Rao. Improving protocol robustness in ad hoc networks through cooperative packet caching and shortest multipath routing, IEEE Transactions on Mobile Computing. 2005; 4(5); 443-457.

[3] Garcia-Luna-Aceves, Jose J. A minimum-hop routing algorithm based on distributed information. Computer Networks and ISDN Systems. 1989; 16(5); 367-382.

[4] Zhang, Xin Ming, En Bo Wang, Jing Jing Xia, Dan Keun Sung. A neighbor coverage-based probabilistic rebroadcast for reducing routing overhead in mobile ad hoc networks. IEEE transactions on mobile computing. $2013 ; 12(3) ; 424-433$.

[5] Ejmaa, Ali Mohamed E, Shamala Subramaniam, Zuriati Ahmad Zukarnain, Zurina Mohd Hanapi. Neighbor-Based Dynamic Connectivity Factor Routing Protocol for Mobile Ad Hoc Network. IEEE Access. 2016; 4; 8053-8064.

[6] Gupta, Sandeep K S, Pradip K, Srimani. Adaptive core selection and migration method for multicast routing in mobile ad hoc networks. IEEE Transactions on Parallel and Distributed Systems. 2003; 14(1); 27-38. 
[7] Shanthi, H J, Anita, E M. Secure and Efficient Distance Effect Routing Algorithm for Mobility (SE_DREAM) in MANETs. In Proceedings of the 3rd International Symposium on Big Data and Cloud Computing Challenges (ISBCC-16'). 2016; 65-80. Springer International Publishing.

[8] Shanthi, H J, Anita, E M. Performance analysis of black hole attacks in geographical routing MANET. 2014.

[9] Adeeb Salh, Lukman Audah, Nor Shahida M. Shah, Shipun A. Hamzah. Maximizing Energy Efficiency for Consumption Circuit Power in Downlink Massive MIMO Wireless Networks. International Journal of Electrical and Computer Engineering (IJECE). 2017; 7(6); 2977 2985. 